\title{
On the Use of Mechanical Filters to Attenuate the Transmission of Tilt Motion to Inertial Sensors
}

\author{
by Fabrice Matichard, ${ }^{*}$ Richard Mittleman, and Matthew Evans
}

\begin{abstract}
Inertial sensors such as seismometers, geophones, and accelerometers cannot distinguish horizontal motion from tilt motion. Rotation measurements can be used to subtract the tilt component from horizontal measurements, but the noise in the tilt sensor is often a limiting factor. No mechanism can change the dual sensitivity of inertial sensors to tilt and translation, but the transmission of ground motion can be mechanically filtered in a frequency-dependent way. This article discusses the use of mechanical filters to reduce the transmission of tilt motion from the ground to inertial instruments, which can be applied to existing sensors, or considered for integration in the design of new horizontal sensors. The limitations of this approach are related to (1) geometrical couplings due to the separation between the reference point and the input point of the mechanical filter, (2) residual tilt transmission through the joints stiffness, (3) effects of the mechanical filtering on the signal-to-noise ratio of the horizontal motion measurement, and (4) practical difficulties with the implementation of such concepts, including thermal noise in the flexures. This study analyzes and quantifies the benefits and limitations of the mechanical filtering approach for seismic studies and for seismic isolation applications.
\end{abstract}

\section{Introduction}

Inertial sensors used to measure translational acceleration are also sensitive to tilt motion as gravity exerts a force along the sensitive axis as a function of the inclination of the instrument. This effect is particularly problematic for horizontal inertial sensors because they are sensitive to this effect to the first order. This problem has been discussed for more than a century in the context of seismological applications. See, for example, Wielandt and Forbriger (1999), Graizer (2005, 2006a,b), Boroschek and Legrand (2006), Forbriger (2006), Lambotte et al. (2006), Kalkan and Graizer (2007a,b), Pillet and Virieux (2007), and Pillet et al. (2009). Inertial sensors are also used in the field of active seismic isolation (Matichard et al., 2015). Tilt-gravity coupling often limits the level of isolation achievable at a low frequency. Lantz et al. (2009) discussed this problem in the context of active seismic isolation for gravitational wave detectors.

There are many ways in which horizontal and tilt motion contributions can be separated by combining multiple instruments. The signals from a pair of vertical sensors can, for example, be combined to estimate the tilt motion. Other methods use rotation sensors which are insensitive to translational acceleration, such as a beam balance whose center of gravity is positioned close to the articulation point. Related discussions and other examples can be found in Bradner and

\footnotetext{
*Also at Caltech, 1200 E California Boulevard, Pasadena, California 91125.
}

Reichle (1973), Wielandt and Forbriger (1999), Forbriger (2009), or Graizer (2009a). When tilt measurements are used to separate the tilt component from a horizontal measurement, the ability to resolve tilt and subtract it is often limited by the self-noise of the instrument used to estimate the tilt motion (Lantz et al., 2009). There are a number of designs of very low-noise rotation sensors which are currently an area of active research, see for instance, Dergachev et al. (2014) or Venkateswara et al. (2014). In Matichard and Evans (2015), the tilt-horizontal coupling problem was discussed in the context of seismological and seismic isolation applications, and various techniques which can be used to resolve tilt and subtract it from a horizontal measurement were reviewed, with particular attention to sensor noise limitations.

As a consequence of the equivalence principle, no mechanism can change the dual sensitivity of the sensor to gravitational and inertial acceleration. However, as discussed above, a rotation of the local reference frame (tilt) can be independently sensed and corrected. Alternatively, mechanical systems can be used to filter the transmission of ground translation and rotation, and change the transmitted ratio in a frequency-dependent way. This article discusses the use of mechanical filters to reduce the transmission of tilt motion from ground to horizontal inertial sensors. The limitations of this approach are related to: 
- the geometrical coupling due to the separation between the reference point and the input point of the mechanical filter (sometimes called angular acceleration coupling);

- the effect of the mechanical filtering on the signal-to-noise ratio (SNR) of the horizontal measurement;

- residual tilt transmission; and

- practical difficulties with the implementation of such concepts.

The goals of this study are to analyze and quantify the benefits and limitations of the mechanical filtering approach. The Tilt-Horizontal Coupling Problem section of the article summarizes the tilt coupling problem. The Motivations and Limitations section presents the motivations and highlights the limitations related to the use of mechanical filters to reduce the transmission of tilt motion to inertial sensors. The Transfer Function from Input Translation to Inertial Sensor Output section studies the transmission of ground translation to suspended instruments and discusses the effects of the mechanical filtering on the sensor noise. The Calculation of the Tilt to Horizontal Translation Ratio section studies the transmission of tilt from ground to the instrument through the mechanical filter. The ratio of the tilt-to-output and translation-to-output transfer functions is studied to estimate the benefits of the mechanical filtering. The Practical Considerations section reviews possible engineering configurations and discusses practical difficulties related to the implementation of such an approach. As an aid to the reader, a list of the notations and definitions is provided in Appendix A.

\section{The Tilt-Horizontal Coupling Problem}

This section describes the tilt-horizontal coupling problem. It defines the notations and assumptions used in this section and those that follow. For more details, a similar case is considered in Graizer (2009b), and before that in Graizer and Kalkan (2008). The problem can be summarized using the model of a horizontal geophone (passive mass-spring seismometer) shown in Figure 1. The reference point is a particular point of interest whose horizontal inertial motion must be measured. It is subjected to input translation $\left(x_{r}\right)$ and input rotation $\left(\theta_{r}\right)$. The inertial sensor is located at a distance $L_{r}$ from the reference point.

For the purpose of this study, the horizontal axis is defined as an axis perpendicular to the local, time-averaged gravity field. It is assumed that the amplitude and direction of gravity can be considered constant over the measurement time. The development and analysis presented in this article hold only if this assumption is a good approximation. Over very long periods, this assumption may not hold.

In this symbolic representation, the inertial instrument is made of a mass mounted on a spring-damper inside a case. The mass provides an inertial reference to measure the inertial motion of the case. The relative motion between the case and the reference mass $\left(\delta_{r}\right)$ will be referred to as the output of the inertial sensor. Although the output signal of geophones

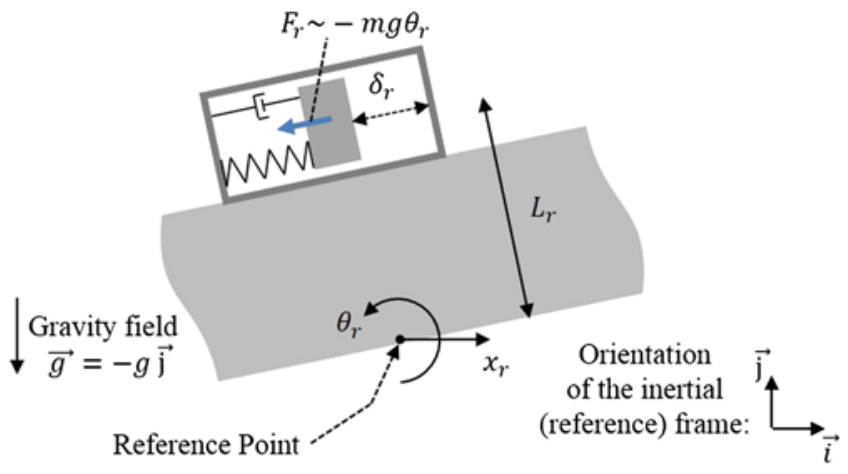

Figure 1. Inertial instrument response to a combination of translational and rotational input motion. The color version of this figure is available only in the electronic edition.

usually measures the first time derivative of $\delta_{r}$, we will analyze $\delta_{r}$ in displacement units. Performing the analysis in velocity units would lead to the same conclusions.

In the following equations and in this article in general, we assume that the motions and angles are such that the sine and tangent of an angle can be approximated by the angle. The study is performed in the Laplace domain ( $s$ is the Laplace variable).

The response of the inertial sensor $\left(\delta_{r}\right)$ is given in equation (1) as a function of the horizontal and tilt motion of the reference point. $H$ is the transfer function of the inertial sensor given in equation (2), in which $\omega$ is the natural frequency of the geophone and $\mu$ is the damping ratio:

$$
\delta_{r}=H\left(s^{2} x_{r}-L_{r} s^{2} \theta_{r}+g \theta_{r}\right),
$$

$$
H=\frac{1}{s^{2}+2 \mu \omega s+\omega^{2}} .
$$

The first three terms in the parenthesis of equation (1) show the relative contribution of the input motions:

- $\left(s^{2} x_{r}\right)$ is the horizontal acceleration. That is the quantity nominally of interest in the measurement.

- $\left(L_{r} s^{2} \theta_{r}\right)$ is the contribution of the angular acceleration of the reference point (geometric coupling).

- $\left(g \theta_{r}\right)$ is the tilt coupling due to gravity, which creates a force $F_{r}$ along the sensing axis of the instrument as shown in Figure 1. Because this term is proportional to the rotation angle while the others are proportional to acceleration, it dominates at low frequency.

The tilt horizontal ratio (THR) is defined as the ratio of the tilt and translation contributions to the readout of the sensor and is given in equation (3). The numerator is the sensitivity of the instrument to the rotation of the reference point, and the denominator is the sensitivity of the instrument to the translation of the reference point:

$$
\mathrm{THR}_{r}=\frac{\partial \delta_{r}}{\partial \theta_{r}} / \frac{\partial \delta_{r}}{\partial x_{r}} .
$$


(a) Differential

Angular

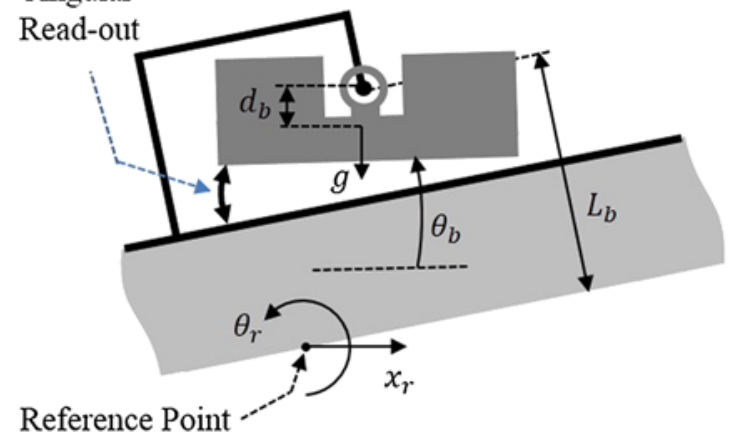

(b)

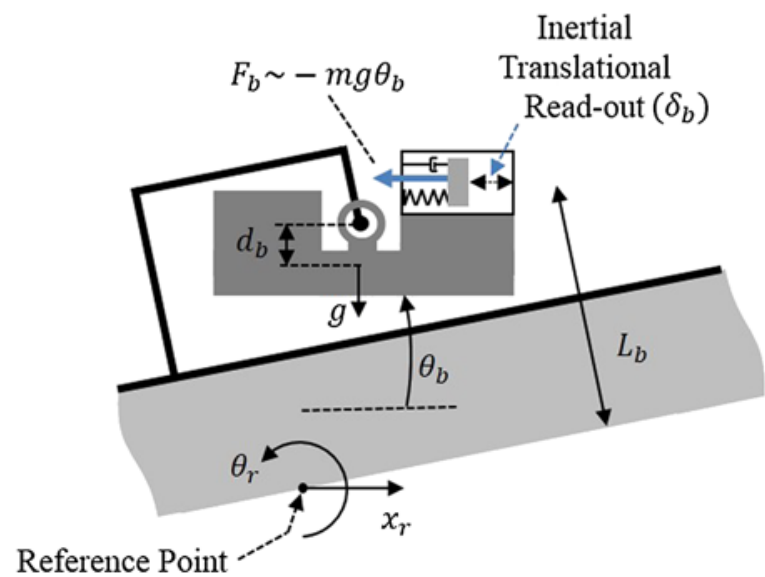

Figure 2. (a) The beam balance is inertially decoupled from ground rotation. (b) Attenuation of the tilt transmission to a horizontal geophone. The color version of this figure is available only in the electronic edition.

Equation (4) combines equations (1)-(3) to give the THR of the ground-fixed instrument. The first term is due to geometrical coupling, the second term is due to tilt-gravity coupling:

$$
\mathrm{THR}_{r}=-L_{r}+\frac{g}{s^{2}} .
$$

In the next sections, the THR of instruments mounted on mechanical filters will be examined to analyze the benefits and limitations of such techniques. For any particular configuration, the lower the ratio, the better the ability to distinguish translation from rotation.

\section{Motivations and Limitations}

In Matichard and Evans (2015), we reviewed subtraction techniques based on the use of multiple instruments to separate horizontal and tilt components, and pointed out limitations related to sensor noise. In this article, we analyze the use of mechanical filters to reduce the transmission of ground tilt to inertial sensors, as an alternative to subtraction techniques. This section presents the motivations for this approach and gives an overview of the benefits and limitations that are analyzed in the following sections.

\section{Motivation}

The use of a mechanical filter to reduce the transmission of ground tilt to horizontal sensors can be seen as the counterpart of the beam balance approach used to measure tilt (shown in Fig. 2a). The beam rotation angle relative to a fixed reference is $\theta_{b}$, and the transfer function from ground rotation to beam rotation is given in equation (5), in which $m_{b}$ is the mass of the beam balance, $I_{b}$ is its moment of inertia, $d_{b}$ is the distance between the articulation point and the center of gravity of the beam, and $k_{b}$ is the stiffness in the joint. Dissipation terms can be added to this model, but they are not necessary for the purpose of this discussion. The natural frequency of the beam balance $\left(\omega_{b}\right)$ is given in equation (6). Assuming the center of gravity is nearly aligned with the articulation point ( $d_{b}$ is small), the natural frequency simplifies to equation (7), and the response of the beam balance simplifies to equation (8). Below the natural frequency, the rotation spring couples the beam balance to the ground motion. Above the natural frequency, the beam rotation is inertially decoupled from the ground rotation. The beam balance can be used as an inertial reference to measure ground tilt with a differential read-out as shown in Figure 2a. This type of sensor is essentially insensitive to horizontal acceleration, as discussed in Venkateswara et al. (2014):

$$
\frac{\theta_{b}}{\theta_{r}}=\frac{k_{b}}{\left(I_{b}+m_{b} d_{b}^{2}\right) s^{2}+m_{b} g d_{b}+k_{b}},
$$

$$
\omega_{b}=\sqrt{\frac{m_{b} g d_{b}+k_{b}}{I_{b}+m_{b} d_{b}^{2}}},
$$

$$
\omega_{b} \sim \sqrt{\frac{k_{b}}{I_{b}}},
$$

and

$$
\frac{\theta_{b}}{\theta_{r}} \sim \frac{\omega_{b}^{2}}{s^{2}+\omega_{b}^{2}} .
$$

In Figure 2b, a geophone is positioned on the beam balance. Assuming that the mass of the inertial instrument is small enough to not affect the transfer function of the beam balance, equations (5)-(8) remain valid (note: the coupling between the moving mass of the instrument and the platform is analyzed in Appendix B). Above the natural frequency, the beam balance is inertially decoupled from the ground, such that its rotation angle $\theta_{b}$ is smaller than the ground rotation angle $\theta_{r}$. The force exerted by gravity on the proof mass of the inertial sensor mounted on the beam balance $\left(F_{b}\right)$ is smaller than in the case of the ground-mounted instrument shown in Figure 1. The effect of gravity through tilt is thus reduced. This can be shown by calculating the THR of this configuration.

The definition of the THR for the inertial sensor mounted on the beam balance $\left(\mathrm{THR}_{\mathrm{b}}\right)$ is given in equation (9). 

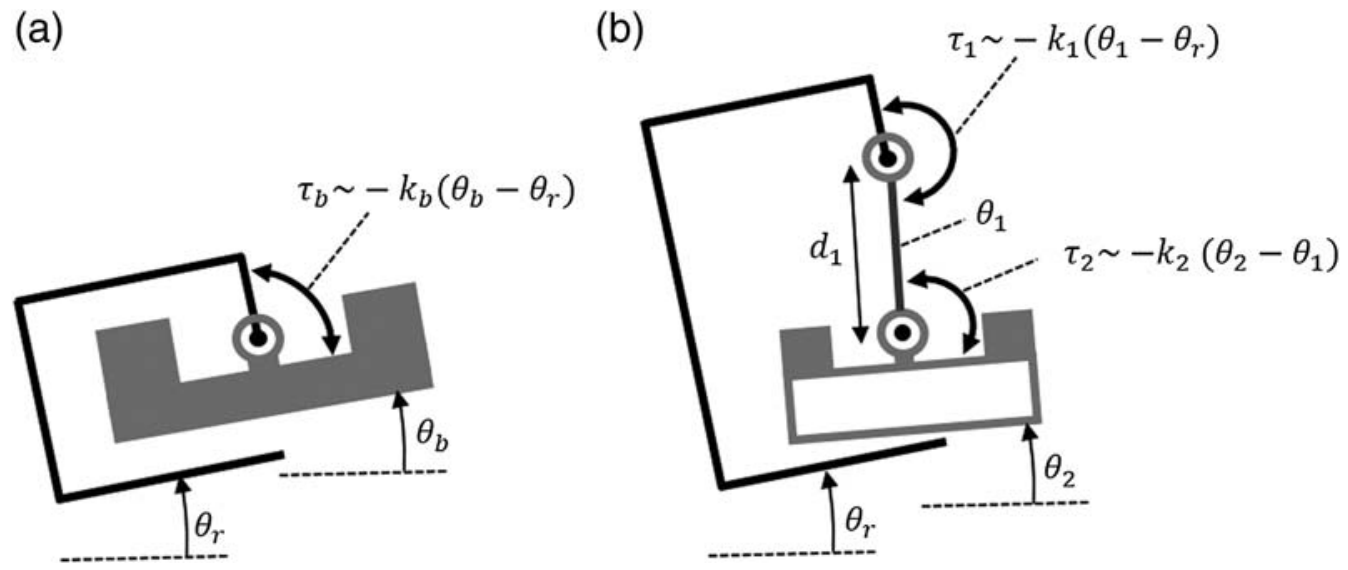

Figure 3. (a) Beam balance and (b) suspension configurations

Assuming that the sensor is aligned with the articulation point, the instrument remains sensitive to linear acceleration $\left(x_{r}\right)$, and geometrical couplings $\left(\theta_{r}\right)$ as given in equation (10), which also includes the tilt gravity coupling (related to $\theta_{b}$ ). Because the beam rotation is mechanically filtered as shown in equation (8), the THR reduces to equation (11). The geometrical coupling is unchanged, but the tilt-gravity coupling is filtered by the term $\omega_{b}^{2} / s^{2}$ above the natural frequency:

$$
\begin{gathered}
\mathrm{THR}_{\mathrm{b}}=\frac{\partial \delta_{b}}{\partial \theta_{r}} / \frac{\partial \delta_{b}}{\partial x_{r}} . \\
\delta_{b}=H s^{2}\left(x_{r}-L_{b} \theta_{r}+\frac{g}{s^{2}} \theta_{b}\right) . \\
\mathrm{THR}_{\mathrm{b}}=-L_{b}+\frac{\omega_{b}^{2}}{\left(s^{2}+\omega_{b}^{2}\right)} \frac{g}{s^{2}} .
\end{gathered}
$$

The goal of this section was to give an example showing how mechanical filters can be used to reduce the contribution of the tilt-gravity coupling. The next sections discuss configurations, benefits, and limitations of such techniques.

Residual Tilt Transmission and Practical Implementation

Below the natural frequency, the beam is not inertially decoupled from ground tilt. The rotation is transmitted through the stiffness of the joint as illustrated in Figure 3a, in which $\tau_{b}$ is the torque in the joint. Above the natural frequency, there is also a residual tilt transmission as shown in equation (11). The tilt transmission can be further attenuated using an intermediate link and joint in a suspension configuration as shown in Figure 3b. The drawback of this configuration is that it reduces the transmission of horizontal motion at higher frequency. This is analyzed in the Transfer Function from Input Translation to Inertial Sensor Output section.
The suspension configuration in Figure $3 \mathrm{~b}$ is also convenient for practical implementation. Flexures can be used to build such linkage (Venkateswara et al., 2014). Metal wires have been used for nearly two decades in suspensions used to isolate the test masses of gravitational wave detectors. In the past decade, silica fibers have been used to build monolithic suspensions with very high-quality factors to reduce thermal noise (Aston et al., 2012). Practical considerations are further discussed in the last section.

This article focuses on the suspension configuration shown in Figure 3b (the configuration in Fig. $3 a$ can be seen as a special case where the length of the intermediate link is zero). The two joints will be called the top and bottom suspension joints, respectively. The intermediate part will be called the suspension link.

\section{Reduction of Horizontal Sensitivity}

One of the drawbacks of the mechanical filtering approach is that it also reduces the sensitivity to horizontal motion. The worst configuration would be a mass-on-spring seismometer mounted on a long and rigid pendulum as shown in Figure 4a. It can be seen as the opposite configuration of the beam balance previously discussed, in which the inertia at the center of gravity $I_{b}$ is now negligible compared with the $m_{b} d_{p}^{2}$ term. As previously discussed, it is assumed that the mass of the inertial sensor does not affect the response of the mechanical filter. This is the worst sensing configuration because the translational and rotational effects of the seismometer mass cancel at the sensor to produce no signal at any frequency, as shown in the following equations.

Equation (12) gives the pendulum response to translation, in which $x_{0}$ is the inertial motion of the input point, and $x_{p}$ is the inertial motion at the center of gravity of the pendulum, in which the inertial sensor is located. Equation (13) gives the angular response to input translation. Equation (14) recalls the geophone response to input translation and input tilt. Equation (15) combines these three equations and shows that the sensor response is zero at all frequencies. In this 

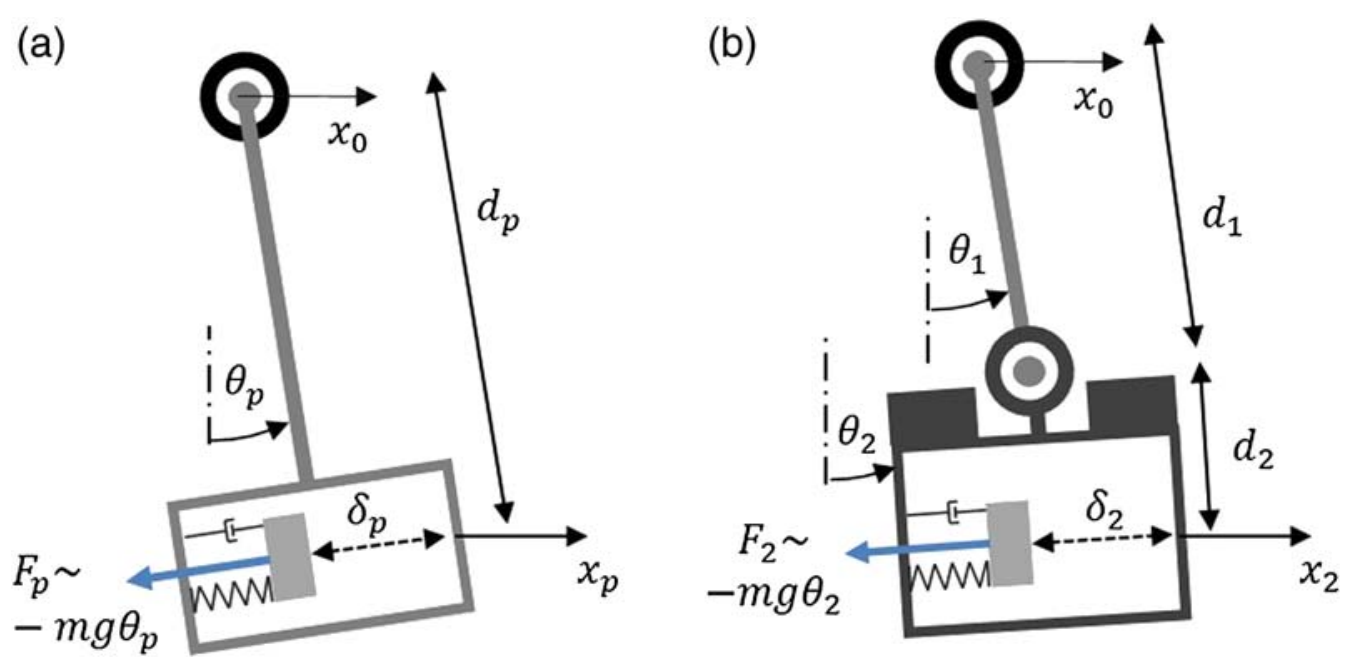

Figure 4. (a) Worst configuration for translation sensitivity. (b) General configuration. The color version of this figure is available only in the electronic edition.

configuration, there is no relative signal between the mass and the case, and therefore there is no output signal:

$$
\begin{gathered}
\frac{x_{p}}{x_{0}}=\frac{g}{\left(d_{p} s^{2}+g\right)}, \\
\frac{\theta_{p}}{x_{0}}=\frac{-s^{2}}{d_{p} s^{2}+g}, \\
\delta_{p}=H\left(s^{2} x_{p}+g \theta_{p}\right), \\
\frac{\delta_{p}}{x_{0}}=0 .
\end{gathered}
$$

In the general case shown in Figure $4 b$, the force due to acceleration $\left(s^{2} x_{2}\right)$ and the force of gravity on the inertial instrument's mass $\left(F_{2}\right)$ do not cancel out at all frequencies. They cancel at DC and at high frequency, but the parameters of the suspension can be chosen to maintain signal $\left(\delta_{2}\right)$ induced by the input motion $\left(x_{0}\right)$ and maintain low THR in a certain frequency band, as detailed in the Transfer Function from Input Translation to Inertial Sensor Output section.

\section{Tilt-Horizontal Ratio}

The mechanical filter, as shown in Figure 5, is used to reduce the tilt transmitted to the sensor. As explained in the previous sections, the filter remains subjected to geometrical coupling and the translation sensitivity is also filtered. The THR from the reference point $\left(x_{r}, \theta_{r}\right)$ to the output of the instrument $\left(\delta_{2}\right)$ is useful to quantify and discuss these effects. The definition of the THR of the suspended instrument is given in equation (16). The goal of this section is to write $\mathrm{THR}_{2}$ as a function of the transfer functions of the mechanical filter:

$$
\mathrm{THR}_{2}=\frac{\partial \delta_{2}}{\partial \theta_{r}} / \frac{\partial \delta_{2}}{\partial x_{r}} .
$$

First, we express the input motion of the mechanical filter $\left(x_{0}, \theta_{0}\right)$ as a function of the motion of the reference point $\left(x_{r}, \theta_{r}\right)$. The rotation of the reference point couples into translation of the input point of the mechanical filter, as shown in Figure 5. Assuming the frame and the local ground are rigid, the rotation of the input point is the same as the rotation of the reference point as given in equation (17). The translation of the suspension point can be written as a function of the translation and rotation of the reference point as given in equation (18):

$$
\begin{gathered}
\theta_{0}=\theta_{r} . \\
x_{0}=x_{r}-L_{0} \theta_{r} .
\end{gathered}
$$

Second, we express the relative motion inside this inertial sensor $\left(\delta_{2}\right)$, which is given in equation (19) as a function of the platform motion $\left(x_{2}, \theta_{2}\right)$. We assume that the inertial sensor is aligned with the center of gravity of the platform (the position of the sensor location does not fundamentally change the results, as discussed in Appendix B):

$$
\delta_{2}=H\left(s^{2} x_{2}+g \theta_{2}\right)
$$

Then, the platform motion is written as a function of the input motion using the mechanical filter transfer functions. The transfer function between the translation at the sensor location and the translation of the input point of the mechanical filter is called $X_{\left(x_{0} \rightarrow x_{2}\right)}$, and the transfer function between the translation at the sensor location and the rotation of the input point is called $X_{\left(\theta_{0} \rightarrow x_{2}\right)}$. These are used to write the translation at the sensor location as a function of the translation and rotation of the input point in equation (20): 


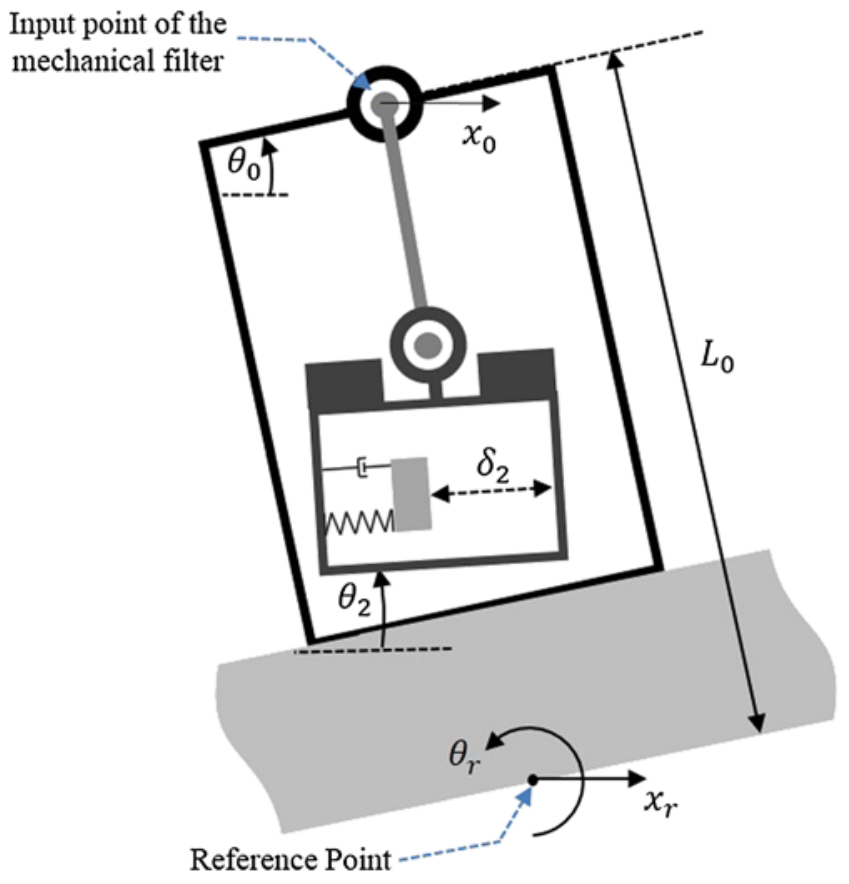

Figure 5. Suspended inertial sensor subjected to the translation and rotation of the reference point. The color version of this figure is available only in the electronic edition.

$$
x_{2}=X_{\left(x_{0} \rightarrow x_{2}\right)} x_{0}+X_{\left(\theta_{0} \rightarrow x_{2}\right)} \theta_{0} .
$$

The transfer function between the platform rotation and the translation of the input point is called $X_{\left(x_{0} \rightarrow \theta_{2}\right)}$, and the transfer function between the platform rotation and the rotation of the input point is called $X_{\left(\theta_{0} \rightarrow \theta_{2}\right)}$. The platform rotation is written as a function of the translation and rotation of the input point in equation (21):

$$
\theta_{2}=X_{\left(x_{0} \rightarrow \theta_{2}\right)} x_{0}+X_{\left(\theta_{0} \rightarrow \theta_{2}\right)} \theta_{0} .
$$

The translation and rotation at the inertial sensor location are expressed as a function of the reference point's translation and rotation in equations (22) and (23), respectively:

$$
\begin{aligned}
& x_{2}=X_{\left(x_{0} \rightarrow x_{2}\right)}\left(x_{r}-L_{0} \theta_{r}\right)+X_{\left(\theta_{0} \rightarrow x_{2}\right)} \theta_{r}, \\
& \theta_{2}=X_{\left(x_{0} \rightarrow \theta_{2}\right)}\left(x_{r}-L_{0} \theta_{r}\right)+X_{\left(\theta_{0} \rightarrow \theta_{2}\right)} \theta_{r} .
\end{aligned}
$$

Finally, the output of the suspended geophone can be written as a function of the motion of the reference point. Equations (19), (22), and (23) are combined in equation (24) to express the internal motion of the suspended inertial sensor as a function of the translation and rotation of the reference point:

$$
\begin{aligned}
\delta_{2}= & H\left[\left(s^{2} X_{\left(x_{0} \rightarrow x_{2}\right)}+g X_{\left(x_{0} \rightarrow \theta_{2}\right)}\right)\left(x_{r}-L_{0} \theta_{r}\right)\right. \\
& \left.+\left(s^{2} X_{\left(\theta_{0} \rightarrow x_{2}\right)}+g X_{\left(\theta_{0} \rightarrow \theta_{2}\right)}\right) \theta_{r}\right] .
\end{aligned}
$$

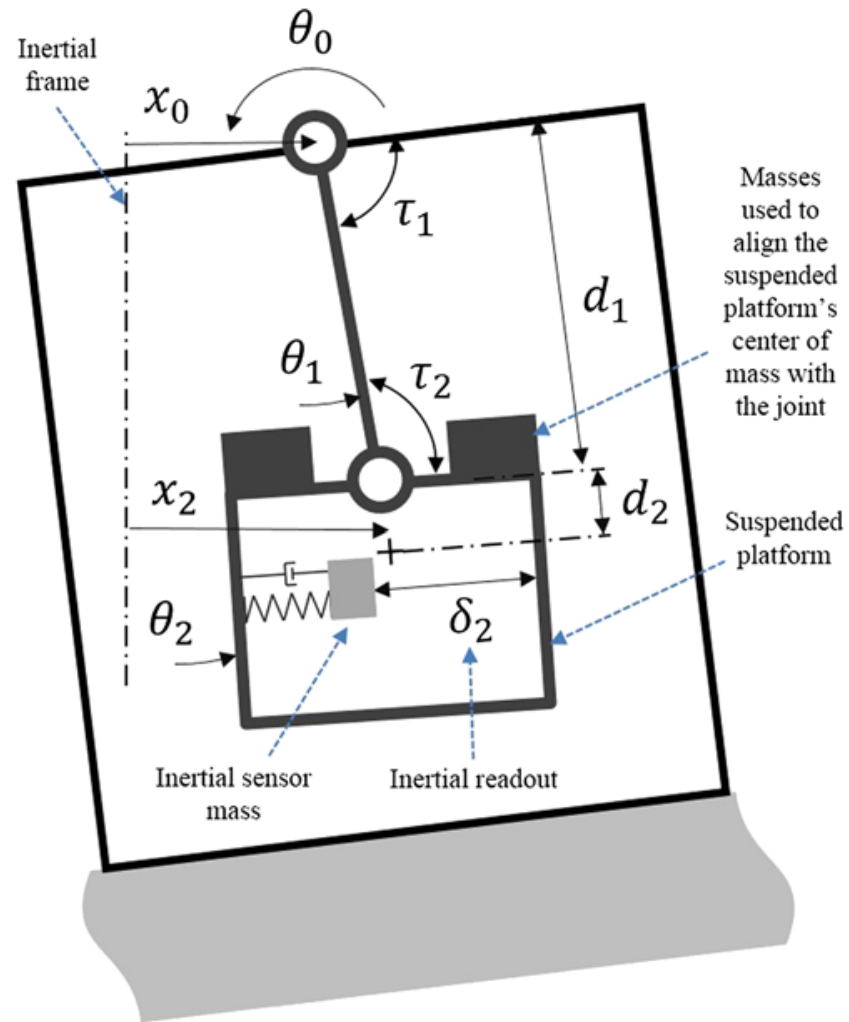

Figure 6. Geometrical parameters. The color version of this figure is available only in the electronic edition.

The tilt-horizontal coupling ratio can then be written as a function of the transfer functions of the mechanical filter as shown in equation (25). The first term $\left(L_{0}\right)$ related to the geometrical coupling is unchanged compared with the reference case described in The Tilt-Horizontal Coupling Problem section. The second term is related to the mechanical filter:

$$
\mathrm{THR}_{2}=-L_{0}+\frac{X_{\left(\theta_{0} \rightarrow x_{2}\right)}+\frac{g}{s^{2}} X_{\left(\theta_{0} \rightarrow \theta_{2}\right)}}{X_{\left(x_{0} \rightarrow x_{2}\right)}+\frac{g}{s^{2}} X_{\left(x_{0} \rightarrow \theta_{2}\right)}} .
$$

In the next sections, transfer functions of the mechanical filter are calculated in order to calculate the THR, and discuss the benefits and limitations of this approach.

\section{Transfer Function from Input Translation to Inertial Sensor Output}

In this section, we analyze the response to translation of an inertial sensor mounted on a mechanical filter as shown in Figure 6. First, we compute the transfer function of the mechanical filter. Then we combine it with the response of the inertial sensor to calculate the transfer function between the input translation and the sensor output. Finally, the effect on the measurement noise is quantified.

\section{Platform's Response to Translation}

Figure 6 shows geometrical parameters of the platform. The mass and moment of inertia of the link are assumed to be 
Table 1

Simulation Parameters

\begin{tabular}{clc}
\hline Symbol & \multicolumn{1}{c}{ Name } & Value \\
\hline$d_{1}$ & Pendulum length & $250 \mathrm{~mm}$ \\
$d_{2}$ & Center of gravity location & $0.1 \mathrm{~mm}$ \\
$m_{2}$ & Suspended mass & $20 \mathrm{~kg}$ \\
$I_{2}$ & Inertia at the center of gravity & $50 \mathrm{~kg} \mathrm{~m}$ \\
$k$ & Joints stiffness & $0.2 \mathrm{~N} \cdot \mathrm{m} / \mathrm{rad}$ \\
$g$ & Acceleration of gravity & $9.81 \mathrm{~m} / \mathrm{s}^{2}$ \\
\hline
\end{tabular}

negligible, as is the mass of the seismometer (the equations of motion including couplings with the proof mass are given in Appendix B). The platform has a mass $m_{2}$ and a moment of inertia $I_{2}$ about its center of gravity. The input motion is $x_{0}$. The angle of the link and the platform, with respect to the vertical direction defined by the gravity field, are $\theta_{1}$ and $\theta_{2}$. The link has a length of $d_{1}$. The displacement of the center of gravity is $x_{2}$. The distance from the bottom joint to the center of gravity of the platform is $d_{2}$.

The motion of the center of gravity is given as a function of the degrees of freedom in equation (26). The torques in the joints are given in equations (27) and (28), in which $\tau_{1}$ is the torque exerted by the input frame on the link and $\tau_{2}$ is the torque exerted by the link on the platform. In this model, we only consider linear stiffness. In practice, the source of these torques can include nonlinear stiffness, viscous damping, and other friction effects depending on the type of joints. Detailed information on the stiffness of flexures can be found in Smith (2000) and Trease et al. (2005). Discussion and references regarding the modeling of friction and damping of pendulums can be found in Peters (2009):

$$
\begin{gathered}
x_{2}=x_{0}+d_{1} \theta_{1}+d_{2} \theta_{2}, \\
\tau_{1}=-k_{1}\left(\theta_{1}-\theta_{0}\right), \\
\tau_{2}=-k_{2}\left(\theta_{2}-\theta_{1}\right) .
\end{gathered}
$$

The sum of the moments on the link can be written as given by equation (29), in which $F_{x}$ is the horizontal force of the link on the platform as given in equation (30), and $F_{z}$ is the vertical force of the link on the platform as given in equation (31). The sum of the moments on the suspended platform produces the angular acceleration given in equation (32). Equations (26)-(32) are combined to produce the equations of motion in equation (33):

$$
\begin{gathered}
\tau_{1}-\tau_{2}-d_{1} F_{x}-\theta_{1} d_{2} F_{z}=0, \\
F_{x}=m_{2} \ddot{x}_{2}, \\
F_{z}=m_{2} g,
\end{gathered}
$$

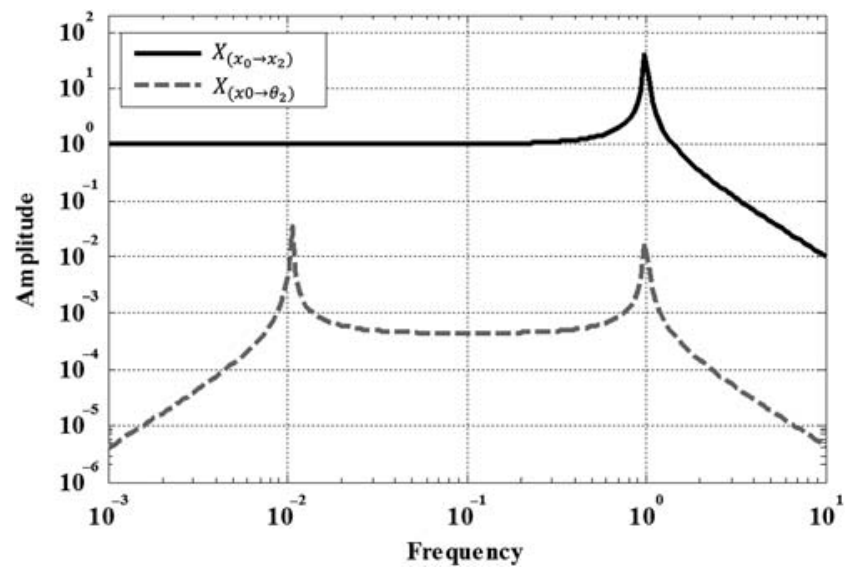

Figure 7. Platform response to translation input.

$$
\tau_{2}-d_{2} F_{x}-d_{2} \theta_{2} F_{z}=I_{2} \ddot{\theta}_{2}
$$

$$
\begin{aligned}
& {\left[\begin{array}{cc}
m_{2} d_{1}^{2} & m_{2} d_{1} d_{2} \\
m_{2} d_{1} d_{2} & I_{2}+m_{2} d_{2}^{2}
\end{array}\right]\left[\begin{array}{l}
\ddot{\theta}_{1} \\
\ddot{\theta}_{2}
\end{array}\right]} \\
& +\left[\begin{array}{cc}
m_{2} g d_{1}+k_{1}+k_{2} & -k_{2} \\
-k_{2} & m_{2} d_{2} g+k_{2}
\end{array}\right]\left[\begin{array}{l}
\theta_{1} \\
\theta_{2}
\end{array}\right] \\
& =\left[\begin{array}{c}
k_{1} \\
0
\end{array}\right] \theta_{0}-\left[\begin{array}{l}
m_{2} d_{1} \\
m_{2} d_{2}
\end{array}\right] \ddot{x}_{0} .
\end{aligned}
$$

The numerical values in Table 1 are chosen to illustrate the response of such a platform. The frequency response is shown in Figure 7, using a structural damping factor of 0.01 in the joints. The solid line shows the transfer function from the input point translation to the inertial sensor location. It is similar to the response of a point mass pendulum. The dashed curve shows the transfer function from the input point translation to the rotation of the platform. It is shaped as a band-pass filter between the natural frequencies.

To explain the response of the platform, the equations of motion can be simplified as follows. Because the center of gravity is positioned close to the bottom joint to reduce the tilt natural frequency, the term $m_{2} d_{2} g$ is small in front of the joint stiffness. We can also assume that the top and bottom joint have the same stiffness $\left(k_{1} \sim k_{2} \sim k\right)$, and that the link is sufficiently long so that the term $m_{2} g d_{1}$ dominates over the joint stiffness. With those assumptions, the equations of motion are reduced to the system given in equation (34). The two natural frequencies can be approximated as shown in equations (35) and (36):

$$
\begin{gathered}
{\left[\begin{array}{ll}
1 & 0 \\
0 & 1
\end{array}\right]\left[\begin{array}{l}
\ddot{\theta}_{1} \\
\ddot{\theta}_{2}
\end{array}\right]+\left[\begin{array}{cc}
\omega_{p} & k /\left(m_{2} d_{1}^{2}\right) \\
-\omega_{t} & \omega_{t}
\end{array}\right]\left[\begin{array}{l}
\theta_{1} \\
\theta_{2}
\end{array}\right]} \\
=\left[\begin{array}{c}
k /\left(m_{2} d_{1}^{2}\right) \\
0
\end{array}\right] \theta_{0}-\left[\begin{array}{c}
1 / d_{1} \\
0
\end{array}\right] \ddot{x}_{0},
\end{gathered}
$$




$$
\begin{aligned}
& \omega_{t} \sim \sqrt{\frac{k}{I_{2}}}, \\
& \omega_{p} \sim \sqrt{\frac{g}{d_{1}}} .
\end{aligned}
$$

The response to the translation input can be approximated as written in equations (37) and (38):

$$
\begin{gathered}
X_{\left(x_{0} \rightarrow x_{2}\right)} \sim \frac{\omega_{p}^{2}}{\left(s^{2}+\omega_{p}^{2}\right)}, \\
X_{\left(x_{0} \rightarrow \theta_{2}\right)} \sim-\frac{1}{d_{1}} \frac{s^{2}}{\left(s^{2}+\omega_{p}^{2}\right)} \frac{\omega_{t}^{2}}{\left(s^{2}+\omega_{t}^{2}\right)} .
\end{gathered}
$$

Below the pendulum frequency, the platform translation follows the ground as shown in equation (39). The platform rotation is a function of the ground acceleration and the tilt filter as shown in equation (40). The next subsection combines the platform's response and the inertial sensor response to calculate the transfer function between the input translation and the sensor output:

$$
\begin{gathered}
s<i \omega_{p} \rightarrow X_{\left(x_{0} \rightarrow x_{2}\right)} \sim 1 \\
s<i \omega_{p} \rightarrow X_{\left(x_{0} \rightarrow \theta_{2}\right)} \sim \frac{-s^{2}}{g} \frac{\omega_{t}^{2}}{\left(s^{2}+\omega_{t}^{2}\right)} .
\end{gathered}
$$

The Translational Response of the Suspended Inertial Sensor

The inertial sensor response written as a function of the suspended platform motion is recalled in equation (41). Equation (42) gives the transfer function between the translation of the input point and the output of the suspended sensor:

$$
\begin{gathered}
\delta_{2}=H\left(s^{2} x_{2}+g \theta_{2}\right), \\
\frac{\delta_{2}}{x_{0}}=s^{2} H\left[X_{\left(x_{0} \rightarrow x_{2}\right)}+\frac{g}{s^{2}} X_{\left(x_{0} \rightarrow \theta_{2}\right)}\right] .
\end{gathered}
$$

Figure 8 shows the frequency response using the parameters defined in Table 1 . The solid curve shows the contribution of the platform translation to the measurement. The dotted curve shows the contribution of the platform rotation. The sum of these two terms is shown by the dashed-dotted curve. It shows that the suspension acts as a mechanical band-pass filter on the transfer function between the input translation and the inertial sensor output.

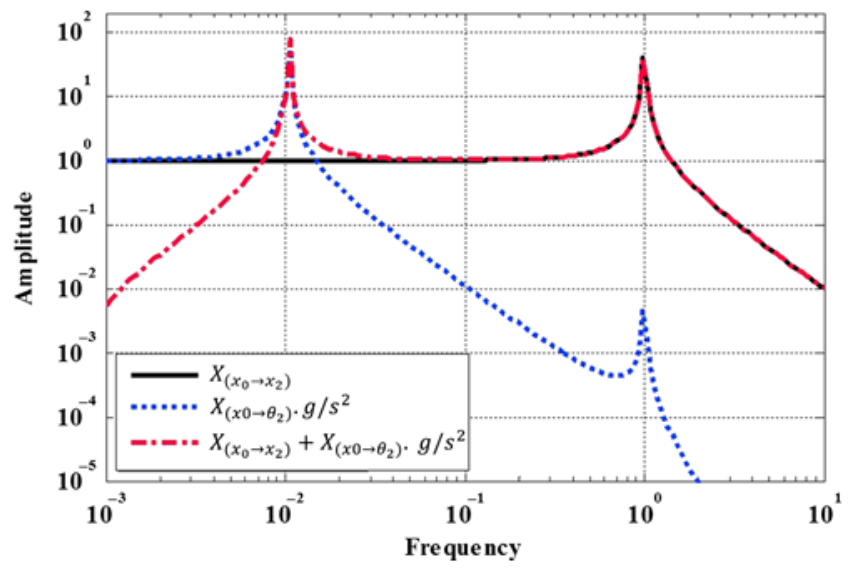

Figure 8. Transfer function from suspension point to inertial sensor's output. The color version of this figure is available only in the electronic edition.

Below the pendulum frequency, this transfer function can be approximated as shown in equation (43). The lowerfrequency sensitivity is inversely proportional to the square of the tilt frequency. Between the resonances, the transfer function is approximately unity. Above the pendulum frequency, the transfer function is filtered by the pendulum response. The higher the pendulum frequency, the better the high-frequency translational sensitivity, but the higher the low-frequency tilt transmission as discussed in the Calculation of the Tilt to Horizontal Translation Ratio section. The next section discusses the effect of the band-pass filtering on the measurement noise:

$$
s<i \omega_{p} \rightarrow X_{\left(x_{0} \rightarrow x_{2}\right)}+\frac{g}{s^{2}} X_{\left(x_{0} \rightarrow \theta_{2}\right)} \sim \frac{s^{2}}{s^{2}+\omega_{t}^{2}} .
$$

\section{Measurement Noise}

The calibrated measurement noise is obtained by combining the inertial sensor self-noise (in $\mathrm{m} / \sqrt{\mathrm{Hz}}$ ) with the transfer function of the suspended sensor as shown in equation (44). The solid curve in Figure 9 shows the noise model of a standard broadband seismometer. The dashed curve shows the noise of the same instrument mounted on a suspension using the parameters defined in Table 1. At all frequencies, the sensor noise is lower than the requirements proposed by Lantz et al. (2009) for rotation sensors to be used for gravitational detectors. It is below the new low-noise model (Peterson, 1993) at all frequencies between $10 \mathrm{mHz}$ and $1 \mathrm{~Hz}$. This plot shows that the loss of signal to noise is acceptable for a large variety of applications, as long as the tilt frequency is sufficiently small. Recent experiments show that such tilt frequencies are achievable, and suitable for robust operations (Venkateswara et al., 2014):

$$
n_{c}=\frac{n}{X_{\left(x_{0} \rightarrow x_{2}\right)}+\frac{g}{s^{2}} X_{\left(x_{0} \rightarrow \theta_{2}\right)}} \text {. }
$$




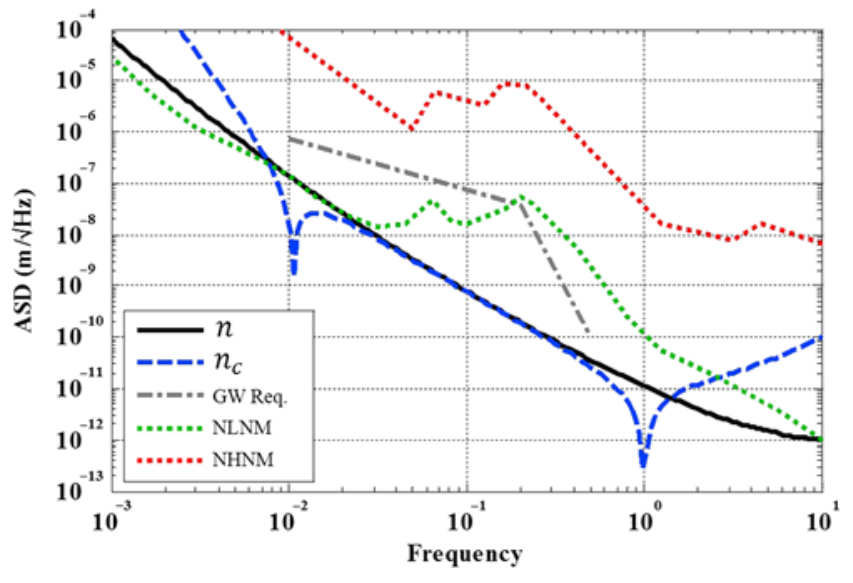

Figure 9. Suspended seismometer calibrated noise. The color version of this figure is available only in the electronic edition.

\section{Calculation of the Tilt to Horizontal Translation Ratio}

This section discusses the influence of the joint stiffness on the tilt transmission, the separation between the reference point and the suspension point, and quantifies the THR. The platform's response to the rotation input can be approximated as written in equations (45) and (46):

$$
\begin{gathered}
X_{\left(\theta_{0} \rightarrow x_{2}\right)} \sim \frac{k}{m_{2} d_{1}} \frac{1}{\left(s^{2}+\omega_{p}^{2}\right)}, \\
X_{\left(\theta_{0} \rightarrow \theta_{2}\right)} \sim \frac{k}{m_{2} d_{1}^{2}} \frac{1}{\left(s^{2}+\omega_{p}^{2}\right)} \frac{\omega_{t}^{2}}{\left(s^{2}+\omega_{t}^{2}\right)} .
\end{gathered}
$$

These transfer functions are combined in equation (47) to approximate the response to input rotation of the sensor mounted on the suspension:

$$
s<i \omega_{p} \rightarrow X_{\left(\theta_{0} \rightarrow x_{2}\right)}+\frac{g}{s^{2}} X_{\left(\theta_{0} \rightarrow \theta_{2}\right)} \sim \frac{k}{m_{2} d_{1} s^{2}} \frac{\omega_{t}^{2}}{\left(s^{2}+\omega_{t}^{2}\right)} .
$$

The formulation given in equation (47) is combined with the system of equations in equation (43) to calculate the THR of the suspended seismometer $\left(\mathrm{THR}_{2}\right)$. The first term is the geometrical coupling, and the second term is the residual tilt transmission through the joints. It is proportional to the square of the stiffness of the joints, and is inversely proportional to the length of the link, the mass, and inertia of the platform:

$$
s<i \omega_{p} \rightarrow \mathrm{THR}_{2} \sim-L_{0}+\frac{k^{2}}{m_{2} d_{1} I_{2} s^{4}} .
$$

Figure 10 compares the THR for the ground-fixed sensor given in equation (4), for the sensor mounted on the beam

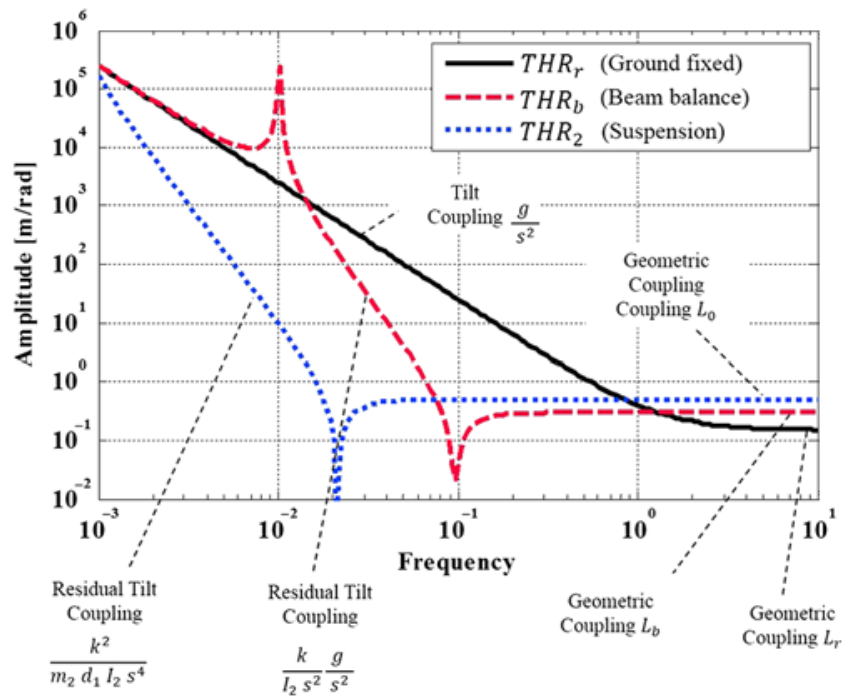

Figure 10. Tilt-horizontal coupling ratio for a seismometer fixed to the ground, a seismometer mounted on a beam balance, and a seismometer mounted on a suspension. The color version of this figure is available only in the electronic edition.

balance given in equation (11) and for the sensor mounted on the suspended platform using equation (33) and the parameters in Table 1.

Realistic values are chosen for the separation between the reference point and the input points: $L_{r}=0.15 \mathrm{~m}$ for the ground-fixed instrument (the distance between the ground surface chosen as reference point, and the center of a broadband sensor); $L_{b}=0.3 \mathrm{~m}$ for the separation between the reference point and the input point of the beam balance (higher than for the ground-fixed instrument to accommodate the geometry of the beam balance); $L_{0}=0.5 \mathrm{~m}$ for the separation between the reference point and the input point of the suspension to account for the length of the intermediate link.

The comparison of the curves in Figure 10 illustrates the benefits of the mechanical filtering approach on the tilt coupling ratio. Although the loss of SNR in some frequency bands pointed out in the previous section are acceptable for many applications, the mechanical filtering of the tilt contribution is a significant benefit in many more applications. The curves in Figure 10 also show the improvements of the suspension configuration in comparison with the beam balance configuration. The intermediate link introduces additional filtering of the tilt-gravity coupling compared with the beam balance configuration. The drawbacks are an increase of the geometrical coupling (unless the reference point and suspension point are aligned), and a loss of SNR at high frequencies induced by the filtering of the translation motion by the pendulum effect. Both of these drawbacks are relatively high-frequency effects $(>1 \mathrm{~Hz})$, and are therefore unimportant for a wide range of applications. The parameters of the suspension, and the length of the link in particular, can be chosen to obtain a suitable compromise. 


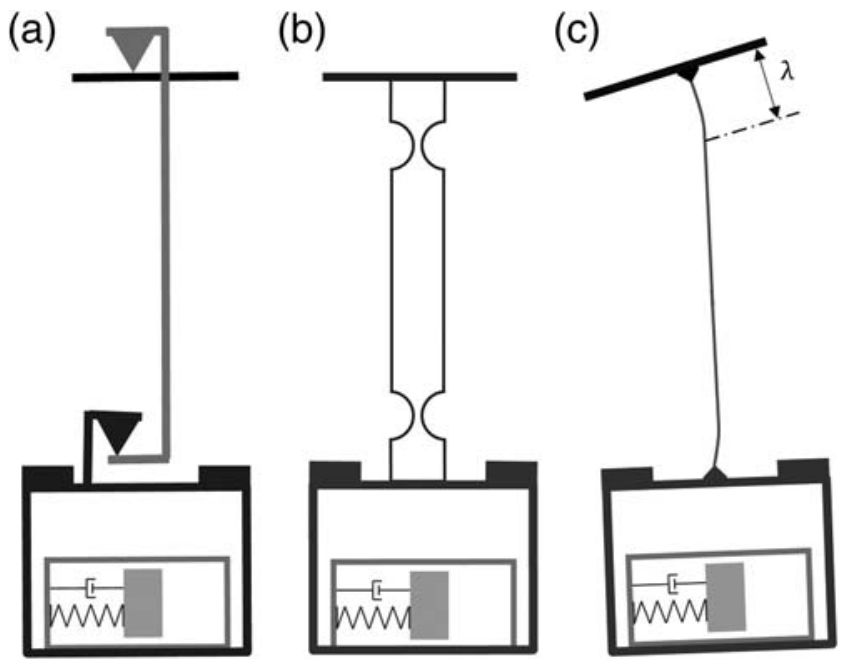

Figure 11. Conceptual representation using (a) knife-edge, (b) flexures, and (c) suspension wires.

\section{Practical Considerations}

There are many ways to implement the mechanical filter approach described in the previous sections. This section reviews some of these configurations and discusses practical considerations relevant to each. Using hinges or bearings to engineer the joints might seem logical, as they match well with the theoretical model of a rotation joint. However, such joints are sometimes not appropriate for engineering systems with small motion because of the friction in the joints. Torques in the joints due to friction can cause strong nonlinear effects in the instrument's response for low-amplitude motions. Knife edges can be used to engineer the joints as illustrated in the conceptual representation in Figure 11a. Useful information related to design and hysteresis effects in knife edges can be found in O'Toole et al. (2014).

Another option for the joints is to use flexible components allowing rotational motion. The use of flexures is a common solution to engineer rotation joints with low loss. An example using a notch type flexure is shown in Figure 11b. Useful information can be found in Smith (2000) or Trease et al. (2005).

Metal wires are a special case of flexure commonly used to engineer suspensions. Silica wires can also be used to reduce the thermal noise (Aston et al., 2012). To first order, a wire suspension can be approximated as illustrated in Figure 11c. The upper and lower part of the wire behave as if pivoted at a distance $\lambda$ from the clamping point. The higher the tension, or the lower the Young's modulus of the wire, the smaller the distance. More details can be found in Cagnoli et al. (2000).

Several suspension configurations can be considered, depending on the objective. A single wire reduces the tilt transmission from the ground to the platform in two directions as illustrated in Figure 12a. Because a high inertia and low wire stiffness are required to filter the tilt, it will also result in a low torsion mode which can produce practical dif- ficulties in the experiment. The problem can be decoupled by using a suspension tuned to filter the tilt in one direction but to remain stiff in other directions. This can be achieved using a ribbon as illustrated in Figure 12b or using a two-wire suspension as in Figure 12c. The latter also allows the instrument to be centered between the wires, which creates a more compact system. The drawback of this approach, relative to a single wire suspension, is that a mechanical filter is needed for each direction of sensing.

To implement any of these concepts, many practical and engineering aspects must be thought through carefully. The tilt and pendulum resonances must be sufficiently damped so as not to compromise the dynamic range of the sensor. Damping can be introduced via the geophone's proof mass as discussed in Appendix B. If a force feedback seismometer is used, little damping will be introduced. Additional damping might be necessary to avoid saturating the sensor, but it must be engineered in a way that does not couple the ground and the platform rotation.

The tilt and pendulum frequencies must be chosen carefully. A low tilt frequency is necessary to maintain the translation sensitivity at low frequencies; however, it creates a higher sensitivity to other low-frequency external disturbances. Systems with very low natural frequencies can be difficult to operate, but recent experiments show that systems with low tilt frequency can operate robustly (Venkateswara et al., 2014).

Similarly, a high pendulum frequency is good for the translation sensitivity, but a short link length may result in higher tilt transmission from the ground to the platform. This is especially true for metal wire suspensions whose bending stiffness increases with the inverse of the wire length.

Moreover, soft suspensions with low resonance frequencies must be carefully shielded from external environmental disturbances such as air currents and temperature fluctuations (Wielandt, 2002; Forbriger et al., 2010). Leveling stability over time must also be considered carefully.

Other issues related to mechanical short-circuits must be considered. For example, the instrument's electrical wires must be soft enough and appropriately routed not to compromise the attenuation of tilt transmission. The effect of the drag force of air on the system should also be carefully studied. References regarding the drag force of air on pendulums can be found in Peters (2009). Furthermore, spring materials must be appropriately chosen to limit the sensitivity to temperature and magnetic fields, and the flexures must be carefully designed to reduce the nonlinearity, hysteresis, and creeping effects.

\section{Conclusion}

The sensitivity of inertial sensors to tilt has been a recurrent problem in seismological studies and seismic isolation applications. In this article, we review the tilt-horizontal coupling problem, and describe a mechanical filter approach which can be used to reduce the transmission of ground tilt 

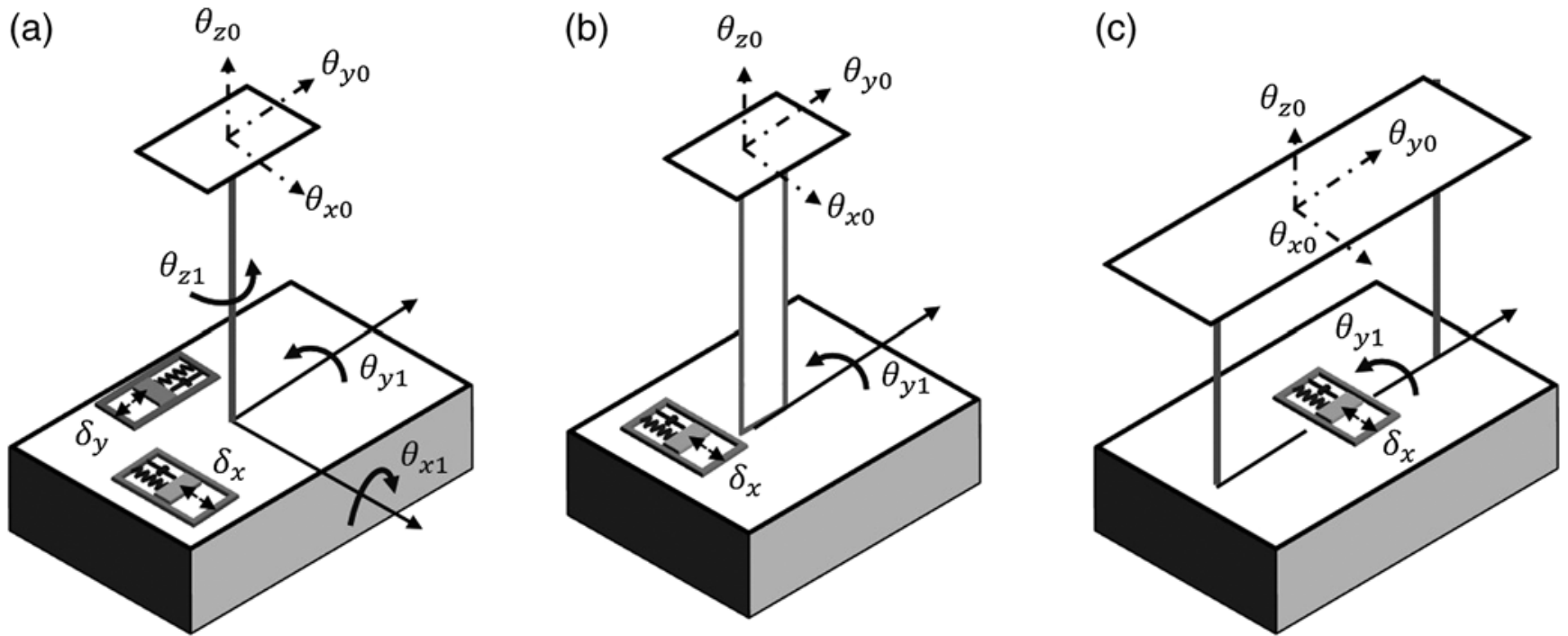

Figure 12. (a) Single-wire, (b) ribbon, and (c) two-wire configuration.

to horizontally mounted inertial sensors. Modeling results show that inertial sensors mounted on this type of mechanical filter remain sensitive to translation in the frequency band between the tilt and the pendulum natural frequencies of the filter, whereas tilt motion is strongly attenuated. The use of suspensions to filter transmission of tilt to inertial sensors is an interesting alternative to tilt subtraction methods, as it does not require a very-low-noise auxiliary tilt sensor. The mechanical filtering approach may be of interest not only for filtering tilt transmission to common seismometers, but also for potential integration in the design of future generations of horizontal inertial sensors.

\section{Data and Resources}

No measurement data were used for this study.

\section{Acknowledgments}

This work was carried out within the LIGO laboratory. LIGO was constructed by the California Institute of Technology and Massachusetts Institute of Technology with funding from the National Science Foundation, and operates under Cooperative Agreement PHY-0757058. Advanced LIGO was built under Award PHY-0823459. This document has been assigned LIGO Laboratory Document Number LIGO-P1400060. The authors are very grateful to Kate Dooley, Krishna Venkateswara, the reviewers, and editors for their comments and suggestions which were very valuable in improving this article.

\section{References}

Aston, S. M., M. A. Barton, A. S. Bell, N. Beveridge, B. Bland, A. J. Brummitt, G. Cagnoli, C. A. Cantley, L. Carbone, A. V. Cumming, et al. (2012). Update on quadruple suspension design for advanced LIGO, Classical Quant. Grav. 29, no. 23, 235,004.
Boroschek, R. L., and D. Legrand (2006). Tilt motion effects on the doubletime integration of linear accelerometers: An experimental approach, Bull. Seismol. Soc. Am. 96, no. 6, 2072-2089.

Bradner, H., and M. Reichle (1973). Some methods for determining acceleration and tilt by use of pendulums and accelerometers, Bull. Seismol. Soc. Am. 63, no. 1, 1-7.

Cagnoli, G., J. Hough, D. DeBra, M. Fejer, E. Gustafson, S. Rowan, and V. Mitrofanov (2000). Damping dilution factor for a pendulum in an interferometric gravitational waves detector, Phys. Lett. A. 272, no. 1, $39-45$.

Dergachev, V., R. DeSalvo, M. Asadoor, A. Bhawal, P. Gong, C. Kim, A. Lottarini, Y. Minenkov, C. Murphy, A. O’Toole, et al. (2014). A high precision, compact electromechanical ground rotation sensor, Rev. Sci. Instrum. 85, no. 5, 054,502.

Forbriger, T. (2008). Low-frequency limit for H/V studies due to tilt, in 67, Jahrestagung der Deutschen Geophysikalischen Gesellschaft (DGG), Aachen, Germany, Extended Abstract.

Forbriger, T. (2009). About the nonunique sensitivity of pendulum seismometers to translational, angular, and centripetal acceleration, Bull. Seismol. Soc. Am. 99, no. 2B, 1343-1351.

Forbriger, T., R. Widmer-Schnidrig, E. Wielandt, M. Hayman, and N. Ackerley (2010). Magnetic field background variations can limit the resolution of seismic broad-band sensors, Geophys. J. Int. 183, no. 1, 303-312.

Graizer, V., and E. Kalkan (2008). Response of pendulums to complex input ground motion, Soil Dynam. Earthq. Eng. 28, 621-631.

Graizer, V. M. (2005). Effect of tilt on strong motion data processing, Soil Dynam. Earthq. Eng. 25, no. 3, 197-204.

Graizer, V. M. (2006a). Tilts in strong ground motion, Bull. Seismol. Soc. Am. 96, no. 6, 2090-2102.

Graizer, V. M. (2006b). Equation of pendulum motion including rotations and its implications to the strong-ground motion, in Earthquake Source Asymmetry, Structural Media and Rotation Effects, Springer, Berlin, Germany, 471-485.

Graizer, V. M. (2009a). Review article: Tutorial on measuring rotations using multipendulum systems, Bull. Seismol. Soc. Am. 99, no. 2B, 1064-1072.

Graizer, V. M. (2009b). The response to complex ground motions of seismometers with Galperin sensor configuration, Bull. Seismol. Soc. Am. 99, no. 2B, 1366-1377.

Kalkan, E., and V. Graizer (2007a). Coupled tilt and translational ground motion response spectra, J. Struct. Eng. 133, no. 5, 609-619.

Kalkan, E., and V. Graizer (2007b). Multi-component ground motion response spectra for coupled horizontal, vertical, angular accelerations and tilt, J. Earthq. Technol. 44, no. 22, 1-33. 
Lambotte, S., L. Rivera, and J. Hinderer (2006). Vertical and horizontal seismometric observations of tides, J. Geodyn. 41, no. 1, 39-58.

Lantz, B., R. Schofield, B. O'Reilly, D. E. Clark, and D. DeBra (2009). Review: Requirements for a ground rotation sensor to improve advanced LIGO, Bull. Seismol. Soc. Am. 99, no. 2B, 980-989.

Matichard, F., and M. Evans (2015) Review: Tilt-free low-noise seismometry, Bull. Seismol. Soc. Am. 105, no. 2A, 497-510.

Matichard, F., B. Lantz, R. Mittleman, K. Mason, J. Kissel, B. Abbott, S. Biscans, J. McIver, R. Abbott, S. Abbott, et al. (2015). Seismic isolation of advanced LIGO: Review of strategy, instrumentation and performance, Classical Quant. Grav. 32, no. 18, 185,003.

O’Toole, A., F. P. Arellano, A. V. Rodionov, M. Shaner, E. Sobacchi, V. Dergachev, R. DeSalvo, M. Asadoor, A. Bhawal, P. Gong, et al. (2014). Design and initial characterization of a compact, ultra high vacuum compatible, low frequency, tilt accelerometer, Rev. Sci. Inst. 85, no. 7, 075003.

Peters, R. D. (2009). Tutorial on gravitational pendulum theory applied to seismic sensing of translation and rotation, Bull. Seismol. Soc. Am. 99, no. 2B, 1050-1063.

Peterson, J. (1993). Observations and modeling of seismic background noise, U.S. Geol. Surv. Open-File Rept. 93-322, 94 pp.

Pillet, R., and J. Virieux (2007). The effects of seismic rotations on inertial sensors, Geophys. J. Int. 171, no. 3, 1314-1323.

Pillet, R., A. Deschamps, D. Legrand, J. Virieux, N. Béthoux, and B. Yates (2009). Interpretation of broadband ocean-bottom seismometer horizontal data seismic background noise, Bull. Seismol. Soc. Am. 99, no. $2 \mathrm{~B}, 1333-1342$.

Smith, S. T. (2000). Flexures: Elements of Elastic Mechanisms, CRC Press, Boca Raton, Florida.

Trease, B. P., Y. M. Moon, and S. Kota (2005). Design of large-displacement compliant joints, J. Mech. Des. 127, no. 4, 788-798.

Venkateswara, K., C. A. Hagedorn, M. D. Turner, T. Arp, and J. H. Gundlach (2014). A high-precision mechanical absolute-rotation sensor, Rev. Sci. Inst. 85, no. 1, 015,005.

Wielandt, E. (2002). Seismic sensors and their calibration, in New Manual of Seismological Observatory Practices, P. Bormann (Editor), International Association of Seismology and Physics of the Earth Interior, 1-46.

Wielandt, E., and T. Forbriger (1999). Near-field seismic displacement and tilt associated with the explosive activity of Stromboli, Ann. Geofisc. 42, no. 3, 407-416.

\section{Appendix A}

\section{List of Notations and Definitions}

Table A1 summarizes the notation used in equations to help follow the course of development in the article.

The goal of the list of definitions is to clarify what we mean by the use of certain words and expressions. We acknowledge that these definitions may differ with the meaning used in other articles and in different communities. The goal is not to provide a universal definition of these terms, but solely to help with clarification in this article.

- Though differentiations of these two terms can be found in the literature, "tilt" and "rotation" are used interchangeably, to avoid repetitions.

- The words "inertial sensor," "geophone," and "seismometer" are used interchangeably to avoid numerous repetitions.

- The word "translation" is often used for "horizontal motion" or "horizontal acceleration".
Table A1

List of Notations

\begin{tabular}{|c|c|c|}
\hline & Variable Description & Symbol \\
\hline \multirow{2}{*}{$\begin{array}{l}\text { General } \\
\text { notations }\end{array}$} & Laplace variable & $s$ \\
\hline & Acceleration of gravity & $g$ \\
\hline \multirow[t]{5}{*}{ Inertial sensor } & Natural frequency of the inertial sensor & $\omega$ \\
\hline & Mass of the inertial sensor & $m$ \\
\hline & Damping ratio of the inertial sensor & $\mu$ \\
\hline & $\begin{array}{l}\text { Mechanical transfer function of the } \\
\text { inertial sensor }\end{array}$ & $H$ \\
\hline & Sensor noise & $n$ \\
\hline \multirow{2}{*}{$\begin{array}{l}\text { Reference } \\
\text { point }\end{array}$} & Translation of the reference point & $x_{r}$ \\
\hline & Rotation angle of the reference point & $\theta_{r}$ \\
\hline \multirow{4}{*}{$\begin{array}{l}\text { Ground-fixed } \\
\text { inertial } \\
\text { sensor }\end{array}$} & $\begin{array}{l}\text { Distance between the reference point } \\
\text { and the sensor }\end{array}$ & $L_{r}$ \\
\hline & $\begin{array}{l}\text { Force along the sensing axis of the } \\
\text { sensor }\end{array}$ & $F_{r}$ \\
\hline & $\begin{array}{l}\text { Relative motion between the case and } \\
\text { the proof mass }\end{array}$ & $\delta_{r}$ \\
\hline & Tilt horizontal ratio & $\mathrm{THR}_{\mathrm{r}}$ \\
\hline \multirow[t]{11}{*}{ Beam balance } & $\begin{array}{l}\text { Distance between the reference point } \\
\text { and the articulation point }\end{array}$ & $L_{b}$ \\
\hline & Mass of the beam balance & $m_{b}$ \\
\hline & Inertia of the beam balance & $I_{b}$ \\
\hline & $\begin{array}{l}\text { Distance between articulation point and } \\
\text { center of gravity }\end{array}$ & $d_{b}$ \\
\hline & Stiffness in the joint & $k_{b}$ \\
\hline & Torque in the joint & $\tau_{b}$ \\
\hline & Rotation angle & $\theta_{b}$ \\
\hline & Natural frequency & $\omega_{b}$ \\
\hline & $\begin{array}{l}\text { Force on the proof mass of the sensor } \\
\text { mounted on the beam balance }\end{array}$ & $F_{b}$ \\
\hline & $\begin{array}{l}\text { Relative motion between the case and } \\
\text { the proof mass }\end{array}$ & $\delta_{b}$ \\
\hline & $\begin{array}{l}\text { Tilt horizontal ratio of the sensor } \\
\text { mounted on the beam balance }\end{array}$ & $\mathrm{THR}_{\mathrm{b}}$ \\
\hline \multirow[t]{6}{*}{ Pendulum } & $\begin{array}{l}\text { Distance between the reference point } \\
\text { and the articulation point }\end{array}$ & $L_{p}$ \\
\hline & Articulation point motion & $x_{0}$ \\
\hline & Pendulum length & $d_{p}$ \\
\hline & Pendulum motion & $x_{p}$ \\
\hline & Rotation angle & $\theta_{p}$ \\
\hline & $\begin{array}{l}\text { Relative motion between the case and } \\
\text { the proof mass }\end{array}$ & $\delta_{p}$ \\
\hline \multirow{11}{*}{$\begin{array}{l}\text { Suspension } \\
\text { filter }\end{array}$} & Top joint motion & $x_{0}$ \\
\hline & Length of the suspension link & $d_{1}$ \\
\hline & $\begin{array}{l}\text { Torque in the top joint of the suspension } \\
\text { filter }\end{array}$ & $\tau_{1}$ \\
\hline & Rotation angle of the suspension link & $\theta_{1}$ \\
\hline & $\begin{array}{l}\text { Distance between bottom joint and the } \\
\text { center of gravity of the platform }\end{array}$ & $d_{2}$ \\
\hline & Mass of the suspended platform & $m_{2}$ \\
\hline & $\begin{array}{l}\text { Moment of inertia about the center of } \\
\text { gravity of the suspended platform }\end{array}$ & $I_{2}$ \\
\hline & Horizontal force exerted by the link & $F_{x}$ \\
\hline & Vertical force exerted by the link & $F_{z}$ \\
\hline & $\begin{array}{l}\text { Torque in the bottom joint of the } \\
\text { suspension filter }\end{array}$ & $\tau_{2}$ \\
\hline & $\begin{array}{l}\text { Motion at the center of gravity of the } \\
\text { suspended platform }\end{array}$ & $x_{2}$ \\
\hline
\end{tabular}

(continued) 
Table A1 (Continued)

\begin{tabular}{|c|c|}
\hline Variable Description & Symbol \\
\hline $\begin{array}{l}\text { Rotation angle of the suspended } \\
\text { platform }\end{array}$ & $\theta_{2}$ \\
\hline $\begin{array}{l}\text { Relative motion between the case and } \\
\text { the proof mass }\end{array}$ & $\delta_{2}$ \\
\hline $\begin{array}{l}\text { Tilt natural frequency of the suspension } \\
\text { filter }\end{array}$ & $\omega_{2}$ \\
\hline $\begin{array}{l}\text { Pendulum natural frequency of the } \\
\text { suspension filter }\end{array}$ & $\omega_{p}$ \\
\hline $\begin{array}{l}\text { Transfer function from input translation } \\
\text { to output translation }\end{array}$ & $X\left(x_{0} \rightarrow x_{2}\right)$ \\
\hline $\begin{array}{l}\text { Transfer function from input rotation to } \\
\text { output translation }\end{array}$ & $X\left(\theta_{0} \rightarrow x_{2}\right)$ \\
\hline $\begin{array}{l}\text { Transfer function from input translation } \\
\text { to output rotation }\end{array}$ & $X\left(x_{0} \rightarrow \theta_{2}\right)$ \\
\hline $\begin{array}{l}\text { Transfer function from input rotation to } \\
\text { output rotation }\end{array}$ & $X\left(\theta_{0} \rightarrow \theta_{2}\right)$ \\
\hline $\begin{array}{l}\text { THR of the sensor mounted on the } \\
\text { suspension filter }\end{array}$ & $\mathrm{THR}_{2}$ \\
\hline
\end{tabular}

- The "reference point" is the origin chosen to describe the body motions.

- "Geometrical coupling" is related to the distance between the reference point and the ground-fixed sensor (or the input point of a mechanical filter), which couples rotation of the ground into translation of the sensor (or the input point of a mechanical filter). This coupling is sometimes also called "angular acceleration coupling."

- "Tilt-gravity coupling" is the effect of gravity on the inertial sensor through tilt.

- "Tilt-horizontal coupling" is the general effect of ground tilt on horizontal sensors. It includes geometrical coupling and tilt-gravity coupling.

- The words sensor "signal" and "output" are used interchangeably. They refer to the "internal motion" of the geophone $(\delta)$, which is the relative motion between the case and the proof mass of the sensor.

- "Tilt-horizontal ratio" (THR), is the ratio of ground tilt $\left(\theta_{r}\right)$ and ground translation $\left(x_{r}\right)$ contributions to the output signal of an inertial sensor. The ground motion is defined at the reference point.

- "Signal subtraction approach" refers to the use of rotational measurements of the ground motion to subtract the tilt component from a horizontal seismometer measurement.

- "Mechanical filtering approach" refers to the use of mechanical systems (beam-balance and suspensions) to filter the transmission of ground tilt and ground translation to an inertial sensor.

\section{Appendix B}

Equations of motion including the couplings between the suspended platform and the geophone's proof mass.

In this article, the coupling between the geophone's proof mass and the platform was assumed to be negligible.
In this section, we model and quantify this effect. A representation of the system is shown in Figure B1.

The input body is labeled (0), the link is labeled (1), the platform is labeled (2), and the instrument's proof mass is called (3). The mass and moment of inertia of the link (1) are assumed negligible. The mass and moment of inertia of the platform (2) are $m_{2}$ and $I_{2}$. The platform includes the geophone's case (fixed parts of the instrument). The mass and moment of inertia of the instrument's proof mass (3) are $m_{3}$ and $I_{3}$. The length of the link is $d_{1}$, the distance between the bottom joint and the center of gravity is $d_{2}$, and the distance between the bottom joint and the instrument is $d_{3}$. The distance between the center of gravity of the platform and the location of the instrument is $d_{\Delta}$. The input displacement is $x_{0}$, the displacement at the bottom joint is $x_{1}$, the displacement at the platform's center of gravity is $x_{2}$, and the displacement of the geophone's proof mass is $x_{3}$. All motions are described with respect to the inertial frame of reference. In equations (B1)-(B3), the motion of the characteristic points is written as a function of the input motion $x_{0}$ and the platform's degrees of freedom $\alpha$ and $\beta$. The terms $\lambda_{0}, \lambda_{1}$, and $\lambda_{2}$ are the transfer functions from the appropriate variable to the proof mass position. They are obtained from the equilibrium of the proof mass (3). They are given in equations (B4)-(B6):

$$
\begin{gathered}
x_{1}=x_{0}+d_{1} \alpha, \\
x_{2}=x_{0}+d_{1} \alpha+d_{2} \beta, \\
x_{3}=\lambda_{0} x_{0}+\lambda_{1} \alpha+\lambda_{2} \beta, \\
\lambda_{0}=\frac{\frac{\omega_{i}}{Q_{i}} s+\omega_{i}^{2}}{s^{2}+\frac{\omega_{i}}{Q_{i}} s+\omega_{i}^{2}}, \\
\lambda_{1}=\frac{d_{1}\left(\frac{\omega_{i}}{Q_{i}} s+\omega_{i}^{2}\right)}{s^{2}+\frac{\omega_{i}}{Q_{i}} s+\omega_{i}^{2}}, \\
\lambda_{2}=\frac{d_{3}\left(\frac{\omega_{i}}{Q_{i}} s+\omega_{i}^{2}\right)-g}{s^{2}+\frac{\omega_{i}}{Q_{i}} s+\omega_{i}^{2}} .
\end{gathered}
$$

Assuming that the damping terms are negligible at low frequencies, equation (B3) shows that for a particular value of $d_{3}$ given in equation (B7), the motion of the proof mass is independent of $\lambda_{2}\left(\lambda_{2} \sim 0\right)$ :

$$
d_{3}=\frac{g}{\omega_{i}^{2}}
$$

The external forces exerted on the link (1) are shown in Figure B1a. Because the mass and inertia of the link are 

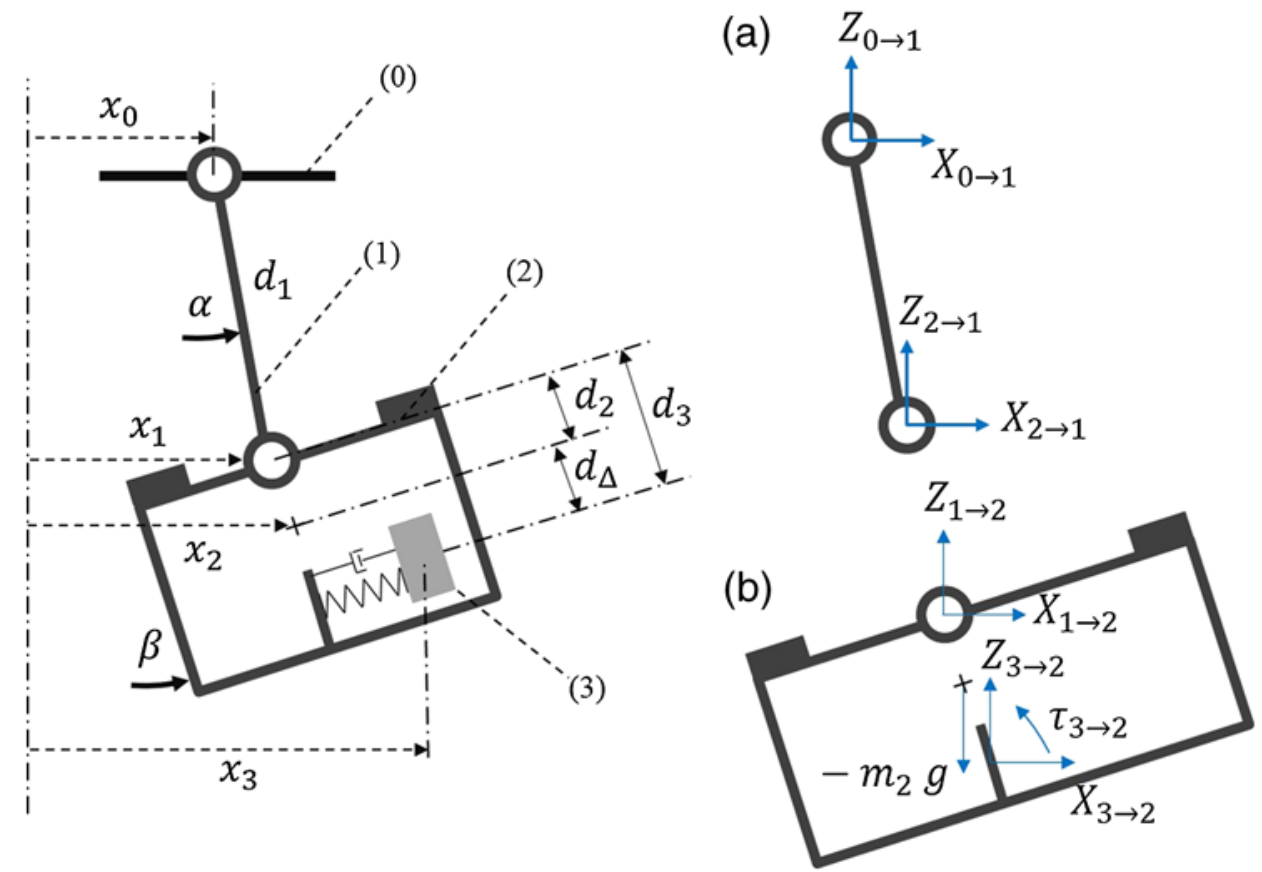

(c) $Z_{2 \rightarrow 3}$

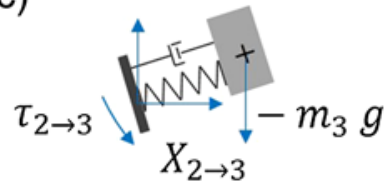

Figure B1. System's geometric parameters. The color version of this figure is available only in the electronic edition.

assumed to be negligible and the joints are assumed to be perfect (no torque in the joints), the equilibrium of the link reduces to the expressions in equations (B8) and (B9), in which $m$ is the total mass of the suspended system (the sum of $m_{2}$ and $m_{3}$ ):

$$
\begin{aligned}
& Z_{2 \rightarrow 1}=-m g, \\
& X_{2 \rightarrow 1}=\alpha m g .
\end{aligned}
$$

The external forces exerted on the platform (2) are shown in Figure B1c. The equilibrium of the body gives the system in equations B10-B12:

$$
\begin{gathered}
\left\{\begin{array}{c}
X_{3 \rightarrow 2} \\
\tau_{3 \rightarrow 2}
\end{array}\right\}=[A]\left\{\begin{array}{l}
\alpha \\
\beta
\end{array}\right\}+[B]\{x\}, \\
A=\left\lceil\quad m_{2} d_{1} s^{2}\right. \\
-m_{2} d_{1} d_{\Delta} s^{2} \\
B=\left[\begin{array}{c}
m_{2} s^{2} \\
-m_{2} d_{\Delta} s^{2}
\end{array}\right] .
\end{gathered}
$$$$
A=\left\lceil\begin{array}{cc}
m_{2} d_{1} s^{2}+m g & m_{2} d_{2} s^{2} \\
-m_{2} d_{1} d_{\Delta} s^{2}-m g d_{3} & I_{2} s^{2}+g\left(m_{2} d_{2}+m_{3} d_{3}\right)-m_{2} d_{2} d_{\Delta} s^{2}
\end{array}\right\rceil,
$$$$
\left\{\begin{array}{c}
X_{2 \rightarrow 3} \\
\tau_{2 \rightarrow 3}
\end{array}\right\}=[C]\left\{\begin{array}{l}
\alpha \\
\beta
\end{array}\right\}+[D] x_{0}
$$$$
C=\left[\begin{array}{cc}
m_{3} \lambda_{1} s^{2} & m_{3} \lambda_{2} s^{2} \\
m_{3} g\left(\lambda_{1}-d_{1}\right) & I_{3} s^{2}+m_{3} g\left(\lambda_{2}-d_{3}\right)
\end{array}\right],
$$$$
D=\left[\begin{array}{c}
m_{3} \lambda_{0} s^{2} \\
m_{3}\left(\lambda_{0}-1\right)
\end{array}\right]
$$

The systems in equations (B10) and (B13) are combined to obtain the system's equations of motion given in equation (B16). The system can be written to express the degrees of freedoms as a function of the input motion as shown in

equation (B17). The response of the suspended instrument is obtained by combining equation (B17) with the instrument response given in equation (B18):

$$
[A]\left\{\begin{array}{l}
\alpha \\
\beta
\end{array}\right\}+[B] x_{0}=-[C]\left\{\begin{array}{l}
\alpha \\
\beta
\end{array}\right\}-[D] x_{0},
$$

The forces applied on the geophone's proof mass (3) are shown in Figure B1b. The equilibrium of the body gives the system in equations (B13)-(B15): 

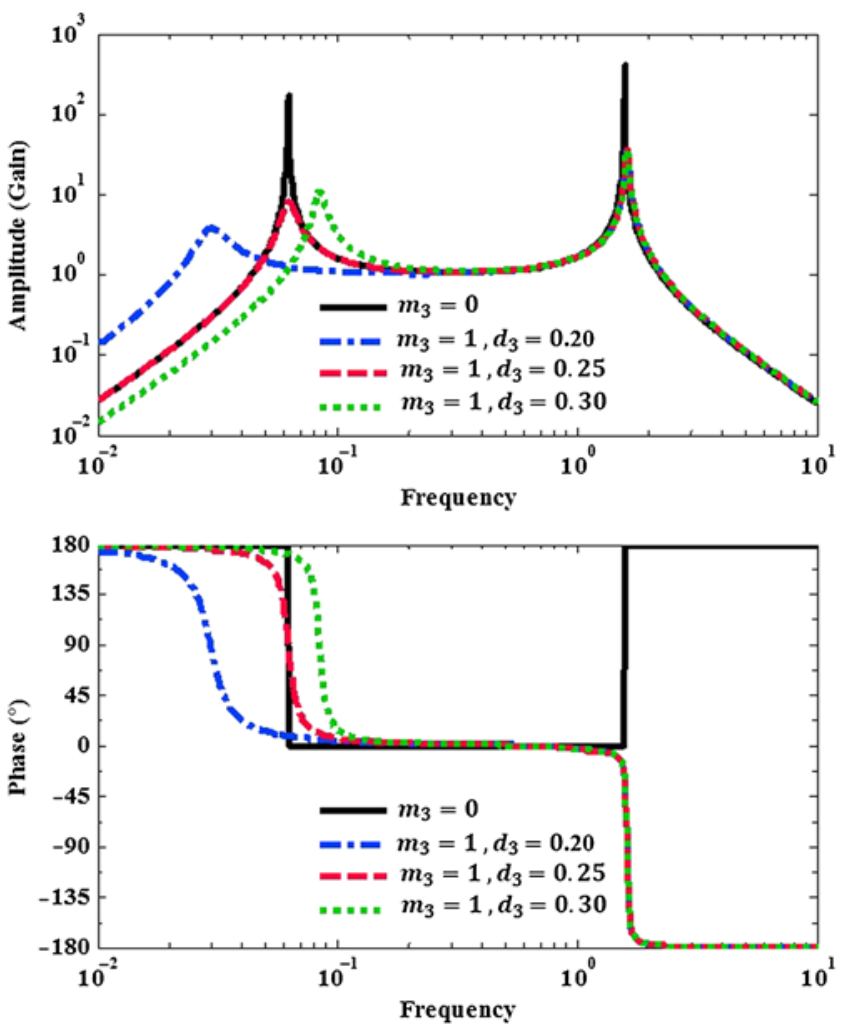

Figure B2. Simulations of the suspended seismometer response $(\hat{x})$ accounting for the motion of the geophone's mass. The color version of this figure is available only in the electronic edition.

$$
\begin{gathered}
\left\{\begin{array}{l}
\alpha \\
\beta
\end{array}\right\}=-[A+C]^{-1}[B+D] x_{0}, \\
\hat{x}=x_{0}+\alpha L+d_{3} \beta+\frac{g}{s^{2}} \beta .
\end{gathered}
$$

Figure B2 shows some simulations of a suspended instrument response $(\hat{x})$ for four specific values of mass and location of the instrument's proof mass. The solid curve is the reference, assuming the proof mass is negligible $\left(m_{3}=0\right)$. The dashed-dotted curve shows the response $\hat{x}$ assuming the mass $m_{3}$ is $1 \mathrm{~kg}$ and the location $d_{3}$ is $0.2 \mathrm{~m}$. The dashed curve shows the response $\hat{x}$ assuming the mass $m_{3}$ is
$1 \mathrm{~kg}$ and the location $d_{3}$ is $0.25 \mathrm{~m}$ The dotted curve shows the response $\hat{x}$ assuming the mass $m_{3}$ is $1 \mathrm{~kg}$ and the location $d_{3}$ is $0.3 \mathrm{~m}$.

The comparison of the black curve with the others highlights that the instrument's internal damping couples with the platform motion at the resonances. The mass and inertia parameters can be tuned to introduce further internal damping, and increase the dynamic range of measurement setup.

The comparison of the curves also illustrates how the instrument's location influences the tilt frequency. For large values of the platform's inertia $\left(I_{2}\right)$ with respect to the proof mass-inertial parameters, the tilt frequency can be approximated as given in equation (B19):

$$
\omega_{t} \sim \sqrt{\frac{m_{2} g d_{2}+m_{3} g\left(d_{3}-\frac{g}{\omega_{i}^{2}}\right)}{I_{2}}} .
$$

The proof mass provides restoring torque to the suspended platform. This restoring torque contains two terms. The first term $\left(m_{3} g d_{3}\right)$ depends on the instrument's vertical location $\left(d_{3}\right)$. The larger this value, the greater the restoring torque and the higher the tilt frequency. The second term $\left(-m_{3} g^{2} / \omega_{i}^{2}\right)$ is related to the proof mass horizontal location. It depends on the spring elongation, which is a function of the instrument's natural frequency $\left(\omega_{i}\right)$. For values of $d_{3}<\left(g / \omega_{i}^{2}\right)$, the geophone's mass lowers the tilt frequency as shown by the dashed-dotted line. For the specific value of $d_{3}=\left(g / \omega_{i}^{2}\right)$, the geophone's mass has no effect on the tilt frequency. For values of $d_{3}>\left(g / \omega_{i}^{2}\right)$, the geophone's mass raises the tilt frequency as shown by the dotted line. This set of simulations illustrates that the mass and location of the instrument influence the tilt frequency of the system but they do not change the shape of the frequency response. The platform still behaves as a mechanical band-pass filter with respect to the translation sensitivity.

LIGO Project MIT

MIT NW22-295

185 Albany Street

Cambridge, Massachusetts 02139

fabrice@ligo.mit.edu

richard@ligo.mit.edu

mevans@ligo.mit.edu

Manuscript received 16 February 2016; Published Online 12 April 2016 